\title{
Photo-Realistic Facial Details Synthesis From Single Image
}

\author{
Anpei Chen ${ }^{1} \quad$ Zhang Chen $^{1}$ \\ Kenny Mitchell ${ }^{2}$ \\ ${ }^{1}$ ShanghaiTech University \\ Guli Zhang ${ }^{1}$ \\ Jingyi $\mathrm{Yu}^{1}$ \\ ${ }^{2}$ Edinburgh Napier University \\ \{chenap, chenzhang, zhanggl,yujingyi\}eshanghaitech.edu.cn K.Mitchell2@napier.ac.uk
}

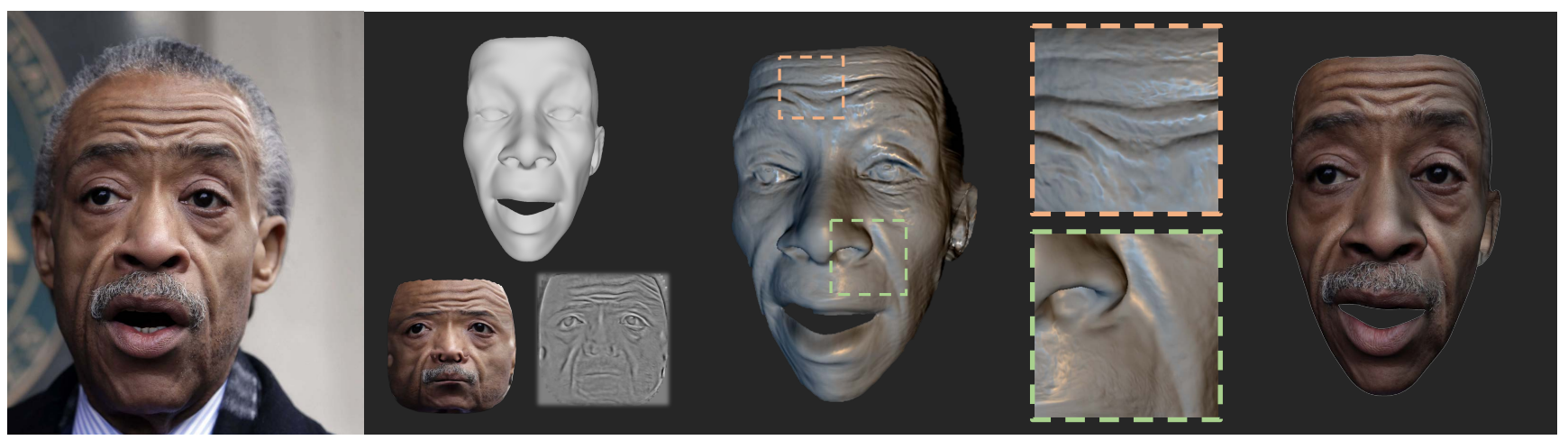

Figure 1. From left to right: input face image; proxy 3D face, texture and displacement map produced by our framework; detailed face geometry with estimated displacement map applied on the proxy 3D face; and re-rendered facial image.

\begin{abstract}
We present a single-image $3 D$ face synthesis technique that can handle challenging facial expressions while recovering fine geometric details. Our technique employs expression analysis for proxy face geometry generation and combines supervised and unsupervised learning for facial detail synthesis. On proxy generation, we conduct emotion prediction to determine a new expression-informed proxy. On detail synthesis, we present a Deep Facial Detail Net (DFDN) based on Conditional Generative Adversarial Net (CGAN) that employs both geometry and appearance loss functions. For geometry, we capture 366 high-quality $3 D$ scans from 122 different subjects under 3 facial expressions. For appearance, we use additional $163 \mathrm{~K}$ in-the-wild face images and apply image-based rendering to accommodate lighting variations. Comprehensive experiments demonstrate that our framework can produce high-quality $3 D$ faces with realistic details under challenging facial expressions.
\end{abstract}

\section{Introduction}

Producing high quality human faces with fine geometric details has been a core research area in computer vision and graphics. Geometric structure details such as wrinkles are important indicators of age and facial expression, and are essential for producing realistic virtual human [2]. Successful solutions by far rely on complex and often expensive capture systems such as stereo-based camera domes [20] or photometric-based LightStage [32, 13]. Although such solutions have become increasingly popular and affordable with the availability of low-cost cameras and lights, they are still bulky and hence do not support portable scanning. In addition, they are vulnerable to low texture regions such as bare skins.

We aim to produce high-quality 3D faces with fine geometric details from a single image, with quality comparable to those produced from the dome systems and LightStage. Existing single-image solutions first construct a 3D proxy face from templates and then refine the proxy by deforming geometry and adding details. Such proxies can be derived from 3D Morphable Model (3DMM) [11, 10, 42, 51, 17] by blending base face geometry. More complex techniques employ sparse coding on 3D face dictionaries to further improve robustness and quality $[41,11,10,42,51,17,23,43$, [8]. However, artifacts arise from these approaches such as over-smoothing and incorrect expression, where a relatively small number of parameters are used to approximate the 
high dimensional space for real face. Shape-from-shading [28], photometric stereo [13], and deep learning [52, 39, 15] have been used to generate the missing details. However, existing methods have limits in attaining correct shape under unseen emotional expressions and lighting, thus delivering insufficient or inaccurate geometric details, as shown in Fig. 7

In this paper, we present a novel learning-based technique to produce accurate geometric details from a single face image. Our approach takes into account emotion, expression and appearance. For proxy generation, we employ the Basel Face Model (BFM) [18] composed of shape, expression and surface reflectance (albedo). 3D expressions, however, exhibit strong ambiguity after being projected onto 2D images: a pair of 3D meshes that represent very different emotional expressions can have similar 2D landmarks on images. Therefore, we first devise a learningbased approach to conduct emotion prediction and then use the result to determine an expression-informed proxy.

For geometric detail synthesis, we devise a Deep Facial Detail Net (DFDN) based on Conditional Generative Adversarial Net (CGAN) to map an image patch to a detailed displacement map. Our DFDN has two components: a medium scale geometry module that learns the PCA coefficients (in our case 64) of each patch and a fine scale geometry module that refines the PCA-based result with additional details. For training, we captured a total of 366 high quality 3D scans from 122 different subjects under three facial expressions (one neutral and two extreme expressions). We augment the training data with 340 high resolution meshes from ICT-3DRFE [47]. The loss function is defined in terms of geometric differences between the estimation and the ground truth. However, we observe that these training data are still insufficient to cover a wide range of lighting conditions. Hence, we introduce an additional unsupervised learning procedure (with an additional 163K images captured in the wild) where for each image we obtain its proxy geometry using our emotion-driven shape estimator and then approximate the corresponding environment lighting using spherical harmonics (SH). We use DFDN to obtain an estimate of the geometry, but since we do not have the ground truth geometry, we re-render these results using the estimated albedo and environment lighting, and compute the loss function in terms of the image differences. Finally, we alternate the supervised and the unsupervised learning processes, on geometry and image, respectively. We have released our code, pre-trained models and results 1

\section{Related Work}

Existing approaches for producing high quality 3D face geometry either rely on reconstruction or synthesis.

1 https://github.com/apchenstu/Facial_Details_ synthesis.git
Reconstruction-based Techniques. Multi-View Stereo (MVS) 3D face reconstruction systems employ stereo [33] or structure-from-motion [56]. A sparse set of cameras produce large scale geometry [20] whereas denser and hence more expensive settings [2] provide more accurate measurements. In either case, the reconstruction quality depends heavily on the feature matching results as they act as anchor points dominating the final shape. For regions with few textures such as bare skin, the reconstruction tends to be overly smooth due to lack of features. For example, wrinkles caused by facial expressions are particularly difficult to reconstruct: even though they cause shading variations, their geometry is too slight to capture using stereo, especially when the camera baseline is small. Recently, Graham et al. [20] use 24 entry-level DSLR photogrammetry cameras and 6 ring flashes to capture facial specular response independently and then combine shape-from-chroma and shape-from-specularity for high quality reconstruction.

Another class of multi-shot techniques employed in face reconstruction is Photometric Stereo (PS). PS is based on analyzing image intensity variations under different illuminations from a fixed viewpoint. Instead of directly reconstructing 3D geometry, PS intends to first recover the normal map and then the 3D mesh, e.g., via normal integration. A common artifact in PS is low-frequency distortions in the final reconstruction [35, 48] caused by perspective projection violating the orthographic assumption. Accurate calibrations on both the light sources and camera, though able to mitigate the problem, are cumbersome. Most recent techniques [36, 57, 16] combine PS with MVS by using the MVS results as a proxy for calibration and then refine the results. Aliaga et al. [3] simulates a MVS setup by employing multiple digital projectors as both light sources and virtual cameras. We refer the readers to [1] for a comprehensive review of PS variants.

Synthesis-based approaches. The availability of high quality mobile cameras and the demand on portable 3D scanning have promoted significant advances on producing high quality $3 \mathrm{D}$ faces from a single image. The seminal work of Blanz and Vetter [6] pre-captures a database of face models and extracts a 3D morphable model (3DMM) composed of base shapes and albedos. Given an input image, it finds the optimal combination of the base models to fit the input. Their technique can also handle geometric deformations under expressions [37, 18] if the database includes expressions, e.g., captured by RGBD cameras [12]. More extensive facial databases have been recently made publicly available [58, 24, 55, 30, 7], with an emphasis on handling complex expressions [30, 7]. Most recently, Li et al. [30] capture pose and articulations of jaw, neck, and eyeballs with over 33,000 3D scans that have helped boost the performance of single-image/video face reconstruction/tracking [41, 11, 10, 46, 60, 42, 51, 17, 23, 43, 8]. 


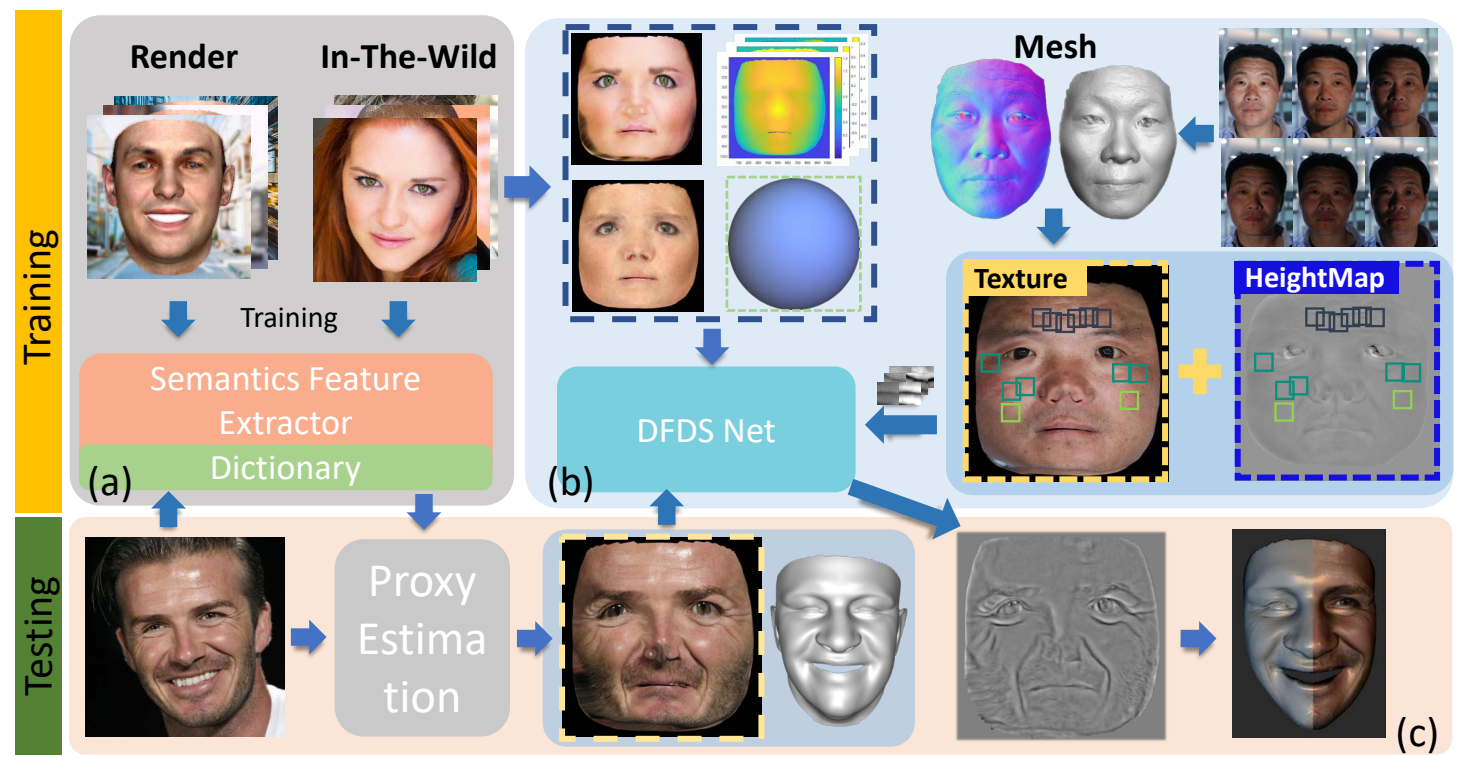

Figure 2. Our processing pipeline. Top: training stage for (a) emotion-driven proxy generation and (b) facial detail synthesis. Bottom: testing stage for an input image.

The current databases, however, still lack mid- and highfrequency geometric details such as wrinkles and pores that are epitomes to realistic 3D faces. Shading based compensations can improve the visual appearance [17, 27] but remain far behind quality reconstruction of photos.

Our approach is part of the latest endeavor that uses learning to recover 3D proxy and synthesize fine geometric details from a single image. For proxy generation, real [53] or synthetically rendered [39, 15] face images are used as training datasets, and convolutional neural networks (CNNs) are then used to estimate 3D model parameters. Zhu et al. [59] use synthesized images in profile views to enable accurate proxy alignment for large poses. Chang et al. [14] bypass landmark detection to better regress for expression parameters. Tewari et al. [50] adopt a selfsupervised approach based on an autoencoder where a novel decoder depicts the image formation process. Kim et al. [29] combine the advantages of both synthetic and real data to [49] jointly learn a parametric face model and a regressor for its corresponding parameters. However, these methods do not exploit emotion information and cannot fully recover expression traits. For detail synthesis, Sela et al. [44] use synthetic images for training but directly recover depth and correspondence maps instead of model parameters. Richardson et al. [40] apply supervised learning to first recover model parameters and then employ shapefrom-shading (SfS) to recover fine details. Li et al. [31] incorporate SfS with albedo prior masks and a depth-image gradients constraint to better preserve facial details. Guo et al. [21] adopt a two-stage network to reconstruct facial geometry at different scales. Tran et al. [54] represent details as bump map and further handle occlusion by hole filling. Learning based techniques can also produce volumetric representations [26] or normal fields [45]. Yet, few approaches can generate very fine geometric details. Cao et.al [9] capture 18 high quality scans and employ a principal component analysis (PCA) model to emulate wrinkles as displacement maps. Huynh et al. [25] use high precision 3D scans from the LightStage [19]. Although effective, their technique assumes similar environment lighting as the LightStage.

\section{Expression-Aware Proxy Generation}

Our first step is to obtain a proxy 3D face with accurate facial expressions. We employ the Basel Face Model $(B F M)$ [18], which consists of three components: shape $M_{\text {sha }}$, expression $M_{\text {exp }}$ and albedo $M_{\text {alb }}$. Shape $M_{\text {sha }}$ and expression $M_{\text {exp }}$ determine vertex positions while albedo $M_{a l b}$ encodes per-vertex albedo:

$$
\begin{aligned}
M_{\text {sha }}(\boldsymbol{\alpha}) & =\boldsymbol{a}_{\text {sha }}+\mathbf{E}_{\text {sha }} \cdot \boldsymbol{\alpha} \\
M_{\text {exp }}(\boldsymbol{\beta}) & =\boldsymbol{a}_{\text {exp }}+\mathbf{E}_{\text {exp }} \cdot \boldsymbol{\beta} \\
M_{a l b}(\boldsymbol{\gamma}) & =\boldsymbol{a}_{a l b}+\mathbf{E}_{a l b} \cdot \boldsymbol{\gamma}
\end{aligned}
$$

where $\boldsymbol{a}_{\text {sha }}, \boldsymbol{a}_{\text {exp }}, \boldsymbol{a}_{\text {alb }} \in \mathbb{R}^{3 n}$ represent the mean of corresponding PCA space. $\mathbf{E}_{\text {sha }} \in \mathbb{R}^{3 n \times 199}, \mathbf{E}_{\text {exp }} \in \mathbb{R}^{3 n \times 100}$ contain basis vectors for shape and expression while $\mathbf{E}_{a l b} \in$ $\mathbb{R}^{3 n \times 199}$ contain basis vectors for albedo. $\boldsymbol{\alpha}, \boldsymbol{\beta}, \boldsymbol{\gamma}$ correspond to the parameters of the PCA model. 


\subsection{Proxy Estimation}

Given a 2D image, we first extract 2D facial landmarks $\mathbf{L} \in \mathbb{R}^{2 m}$ and use the results to compute PCA parameters $\boldsymbol{\alpha}, \boldsymbol{\beta}$ for estimation of proxy shape. Specifically, we set out to find the parameters that minimize the reprojection error on landmarks:

$$
E=\sum_{k} w_{k}\left\|\mathbf{L}_{k}-P\left(\mathbf{l}_{k}(\boldsymbol{\alpha}, \boldsymbol{\beta})\right)\right\|_{2}+\lambda_{s}\|\boldsymbol{\alpha}\|_{2}
$$

where $\mathbf{l}_{k}(\boldsymbol{\alpha}, \boldsymbol{\beta})$ corresponds to the $k$ th facial vertex landmark and $P(\cdot)$ is the camera projection operator that maps $3 \mathrm{D}$ vertices to $2 \mathrm{D}$ image coordinates. $w_{k}$ controls the weight for each facial landmark whereas $\lambda_{s}$ imposes regularization on the shape parameters.

To solve for Eq. 4, we use the iterative linear method in [24]. Specifically, the camera projection operator $P(\cdot)$ is parameterized as an affine camera matrix. For expression parameters $\boldsymbol{\beta}$, different from [24], we fix it as prior parameters $\boldsymbol{\beta}_{\text {prior }}$ computed in Section 3.2. During each round of iterations, we first fix $\boldsymbol{\alpha}$ and solve for $P(\cdot)$ using the Gold Standard Algorithm [22]. We then fix $P(\cdot)$ and solve for $\boldsymbol{\alpha}$. To bootstrap this iterative scheme, we initialize $\boldsymbol{\alpha}$ as $\mathbf{0 .}$

\subsection{Imposing Expression as Priors}

The most challenging component in proxy estimation is expression. 3D expressions exhibit a significant ambiguity after being projected onto 2D images, e.g., different expressions may have similar 2D facial landmarks after projection. Fig. 3 shows an example of this ambiguity: the landmarks of the two faces are extremely close to each other while their expression parameters and shapes are vastly different, especially around nasolabial folds. So it is hard to define or train a mapping directly from image to $3 \mathrm{D}$ expression. In our experiments, we also observe that the reprojectionbased loss function can easily fall into local minimum that reflects such ambiguity.

We propose to use facial semantic information to narrow the proxy parameter solving space via converting the problem into a conditional distribution. Our high level semantic features comprise of emotion features and physically-based appearance features (e.g. FACS).

To obtain emotion features, we reuse AffectNet dataset [34] to train an emotion feature predictor EmotionNet. The dataset contains 11 discrete emotion categories and about $450 \mathrm{~K}$ annotated images. We utilize the "sequential fully-CNN" [4] architecture to train our emotion feature predictor and use the output of the second last layer $f \in \mathbb{R}^{128}$ as the feature vector to represent human emotions. More details could be found in our released code. Next, we randomly generate expression parameters $\boldsymbol{\beta}$ from normal distribution in interval $[-3,3]$ and render $90 \mathrm{~K}$ images with different facial expressions. We feed the images into the

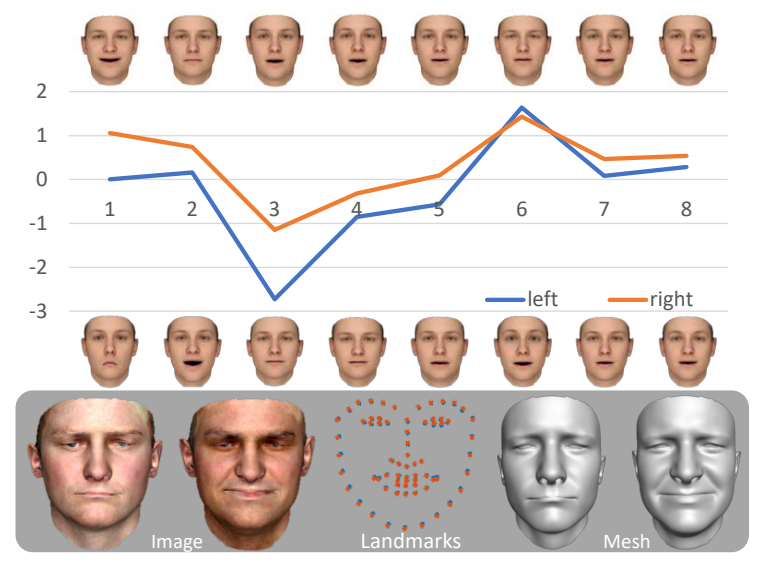

Figure 3. Expression projection ambiguity. Top: Visualization of the two models' first eight dimensions of 3D expression parameters. Bottom: Rendered 2D facial images, their landmarks layered onto each other, and their corresponding meshes. 'left, right' refer to both rendered images and meshes.

trained Emotion-Net and obtain a total of 90K emotion feature vectors. We also use [5] to estimate $90 \mathrm{~K}$ appearance feature vectors on the images. Concatenating these emotion feature vectors along with their corresponding appearance feature vectors, we obtain the semantic feature vector for each of the $90 \mathrm{~K}$ images. We then formulate a dictionary $\Psi: \Psi_{\text {sem }} \rightarrow \Psi_{\text {exp }}$ that record the mapping from semantic features $\Psi_{\text {sem }}$ to expression parameters $\Psi_{\text {exp }}$. Once we obtain the trained model and the expression dictionary, we can predict expression parameters $\boldsymbol{\beta}_{\text {prior }}$ as a prior for proxy estimation.

Given a new image $I$, we first feed it to Emotion-Net and appearance feature predictor to obtain its semantic feature vector. We then find its closest semantic feature vector in the dictionary and use the corresponding expression parameters for $\boldsymbol{\beta}_{\text {prior }}$ :

$$
\left.\boldsymbol{\beta}_{\text {prior }}=\Psi \underset{\psi_{\text {sem }}}{\arg \min } \| \text { Emotion-Net }(I)-\psi_{\text {sem }} \|_{2}\right)
$$

\section{Deep Facial Detail Synthesis}

With the 3D proxy face, we synthesize geometric details by estimating displacement map and applying to the proxy mesh. The key observation here is that for facial details such as wrinkles, there is strong correlation between geometry and appearance.

\subsection{Network Architecture}

Fig. 4 shows our Deep Facial Detail Net (DFDN) with two main cascaded modules. The Partial Detail Inference Module (PDIM) takes 2D image patches as inputs and generates 3D facial geometric details using a PCA-based technique (Section 4.2). Such a scheme dramatically reduces 


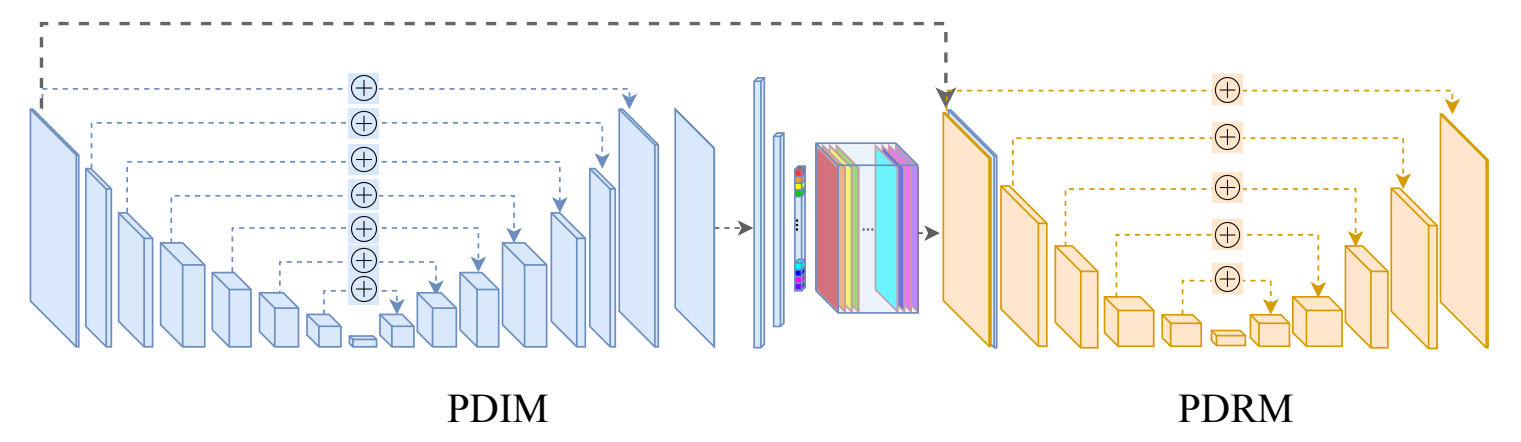

Figure 4. Network architecture for facial detail synthesis. PDIM for medium-frequency detail (wrinkles) synthesis and PDRM for highfrequency detail (pores) synthesis.

the parameter space and is stable for both training and inference process. However, PCA-based approximations lose high-frequency features that are critical to fine detail synthesis. We therefore introduce the Partial Detail Refinement Module (PDRM) to further refine high-frequency details. The reason we explicitly break down facial inference procedure into linear approximation and non-linear refinement is that facial details consist of both regular patterns like wrinkles and characteristic features such as pores and spots. By using a two-step scheme, we encode such priors into our network.

In PDIM module, we use UNet-8 structure concatenated with 4 fully connected layers to learn the mapping from texture map to PCA representation of displacement map. The sizes of the 4 fully connected layers are 2048, 1024, 512, 64 . Except for the last fully connected layer, each linear layer is followed by an $R e L U$ activation layer. In the subsequent PDRM module, we use UNet-6, i.e. 6 layers of convolution and deconvolution, each of which uses $4 \times 4$ kernel, 2 for stride size, and 1 for padding size. Apart from this, we adopt LeakReLU activation layer except for the last convolution layer and then employ tanh activation.

To train PDIM and PDRM modules, we combine supervised and unsupervised training techniques based on Conditional Generative Adversarial Nets (CGAN), aiming to handle variations in facial texture, illumination, pose and expression. Specifically, we collect 706 high precision 3D human faces and over $163 \mathrm{~K}$ unlabeled facial images captured in-the-wild to learn a mapping from the observed image $x$ and the random noise vector $z$ to the target displacement map $y$ by minimizing the generator objective $G$ and maximizing $\log$-probability of 'fooling' discriminator $D$ as:

$$
\min _{G} \max _{D}\left(\mathcal{L}_{c G A N(G, D)}+\lambda \mathcal{L}_{L 1(G)}\right)
$$

where we set $\lambda=100$ in all our experiments and

$$
\begin{aligned}
\mathcal{L}_{c G A N(G, D)}= & \mathbb{E}_{x, y}[\log D(x, y)]+ \\
& \mathbb{E}_{x, z}[\log (1-D(x, G(x, z)))] .
\end{aligned}
$$

A major drawback of the supervised learning scheme mentioned above is that the training data, captured under fixed setting (controlled lighting, expression, etc.), are insufficient to emulate real face images that exhibit strong variations caused by environment lighting and expressions. We hence devise a semi-supervised generator $G$, exploiting labeled 3D face scans for supervised loss $\mathcal{L}_{\text {scans }}$ as well as image-based modeling and rendering for unsupervised reconstruction loss $\mathcal{L}_{\text {recon }}$ as:

$$
\mathcal{L}_{L 1(G)}=\mathcal{L}_{\text {scans }}(x, z, y)+\eta \mathcal{L}_{\text {recon }}(x)
$$

where $x$ is input image, $z$ is random noise vector and $y$ is groundtruth displacement map. $\eta$ controls the contribution of reconstruction loss and we fix it as 0.5 in our case. In the following subsections, we discuss how to construct the supervised loss $\mathcal{L}_{\text {scans }}$ for geometry and unsupervised loss $\mathcal{L}_{\text {recon }}$ for appearance.

\subsection{Geometry Loss}

The geometry loss compares the estimated displacement map with ground truth. To do so, we need to capture ground truth facial geometry with fine details.

Face Scan Capture. To acquire training datasets, we implement a small-scale facial capture system similar to [13] and further enhance photometric stereo with multiview stereo: the former can produce high quality local details but is subject to global deformation whereas the latter shows good performance on low frequency geometry and can effectively correct deformation.

Our capture system contains 5 Cannon 760D DSLRs and 9 polarized flash lights. We capture a total of 23 images for each scan, with uniform illumination from 5 different viewpoints and 9 pairs of vertically polarized lighting images (only from the central viewpoint). The complete acquisition process only lasts about two seconds. For mesh reconstruction, we first apply multi-view reconstruction on the $5 \mathrm{im}$ ages with uniform illumination. We then extract the specular/diffuse components from the remaining image pairs and 
calculate diffuse/specular normal maps respectively using photometric stereo. The multi-view stereo results serve as a depth prior $z_{0}$ for normal integration [38] in photometric stereo as:

$$
\begin{array}{r}
\min \iint_{(u, v) \in I}\left[\left(\nabla z(u, v)-[p(u, v), q(u, v)]^{\top}\right)^{2}\right. \\
\left.+\mu\left(z(u, v)-z_{0}(u, v)\right)^{2}\right] d u d v
\end{array}
$$

where $u, v$ represents image coordinates, $p, q$ represents approximations to $\partial_{u} z$ and $\partial_{v} z$ respectively, $z_{0}$ is the depth prior. $\mu$ controls the contribution of prior depth $z_{0}$. In order to generate geometry pairs with and without details, we set the weight parameter $\mu$ to $1 e^{-5}$ and $1 e^{-3}$ respectively. Then we obtain a ground truth displacement map for each geometry pair.

PCA-Based Displacement Map. In our training scheme, we choose not to directly feed complete face images as inputs to the network: such training can easily cause overfitting since we do not have sufficient 3D face models with fine details to start with. Instead, we observe that despite large scale variations on different faces, local texture details present strong similarities even if the faces appear vastly different. Hence we adopt the idea from [9, 13] to enhance our network generalization by training the network with texture/displacement patches of $256 \times 256$ resolution. We model the displacement using PCA, where each patch is a linear combination of 64 basis patches.

Our geometric loss is then defined as:

$$
\begin{aligned}
\mathcal{L}_{\text {scans }}(x, z, y) & =\sum\|\mathcal{P C} \mathcal{A}(\mathcal{P D} \mathcal{I} \mathcal{M}(x, z))-y\|_{1}+ \\
& \|\mathcal{P} \mathcal{D} \mathcal{R} \mathcal{M}(\mathcal{P C} \mathcal{A}(\mathcal{P} \mathcal{D} \mathcal{I} M(x, z)))-y\|_{1},
\end{aligned}
$$

where $\mathcal{P C A}(\cdot)$ uses input PCA coefficients to linearly combine basis patches. By using the geometry loss, we combine the loss in PCA space with the per-pixel loss to recover finer details.

For patch sampling, we unfold each facial image into a $2048 \times 2048$ resolution texture map and regionally sample training patches based on semantic facial segmentation. For the sampling scheme, we iteratively reduce the displacement map gradient with a weighted Gaussian kernel for training set while we uniformly sample patches with 50\% overlap during inference.

\subsection{Appearance Loss}

Recall that the small amount of labeled facial geometry is insufficient to cover a broad range of illumination conditions and surface reflectance. Thus, we further adopt a rendering-based, unsupervised learning approach: we obtain $163 \mathrm{~K}$ in-the-wild images, estimate its proxy (using the approach in Section 3.2) and geometric details (using

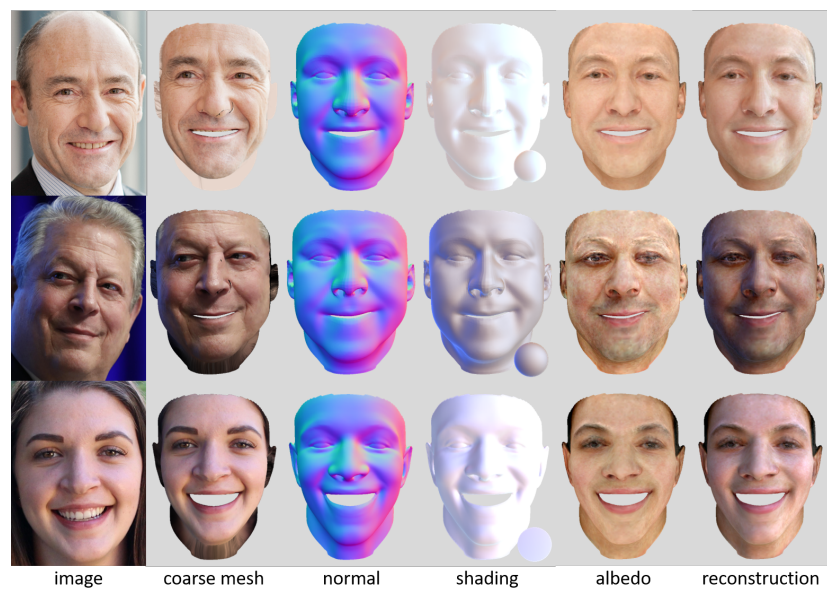

Figure 5. From left to right: from input image, we estimate proxy mesh, normal, lighting/shading, and albedo to re-render an image.

$D F D N$ ), and then use this information to calculate lighting and albedo. Finally, we re-render an image with all these estimations and compute reconstruction loss against the input image during training.

To obtain per-pixel normals with geometric details added, we propose an texture space manipulation using the proxy mesh's position map $\mathcal{P}_{\text {proxy }}$ (shown in Fig. 2 the middle of first row) and the output displacement map $G(u, v)$ from $D F D N$ :

$$
\begin{gathered}
\mathcal{P}_{\text {fine }}(u, v)=\mathcal{P}_{\text {proxy }}(u, v)+G(u, v) \cdot \mathcal{N}_{\text {proxy }}(u, v) \\
\mathcal{N}_{\text {fine }}=\mathcal{F}\left(\mathcal{P}_{\text {fine }}\right)
\end{gathered}
$$

where $\mathcal{N}_{\text {proxy }}, \mathcal{N}_{\text {fine }}$ represent normal map of proxy and fine scale geometry, and $\mathcal{P}_{\text {fine }}$ is the position map of detailed mesh. $\mathcal{N}_{\text {proxy }}, \mathcal{P}_{\text {proxy }}$ are pre-rendered by a traditional rasterization rendering pipeline.

$\mathcal{F}(\cdot)$ is normalized cross product operator on position difference:

$$
\mathcal{F}\left(\mathcal{P}_{\text {fine }}\right)=\frac{\operatorname{conv}_{h}\left(\mathcal{P}_{\text {fine }}\right) \times \operatorname{conv}_{v}\left(\mathcal{P}_{\text {fine }}\right)}{\left\|\operatorname{conv}_{h}\left(\mathcal{P}_{\text {fine }}\right)\right\| \cdot \| \operatorname{conv}_{v}\left(\mathcal{P}_{\text {fine }} \|\right.}
$$

We compute position difference via nearby horizontal and vertical 3 pixels in texture space, giving rise to convolution kernels of $[-0.5,0,0.5]$ and $[-0.5,0,0.5]^{\top}$ for conv $_{h}$, $\operatorname{conv}_{v}$ respectively.

To reconstruct the appearance loss, we assume a Lambertian skin reflectance model and represent the global illumination using spherical harmonics (SH) to estimate environment lighting $S$ and albedo $\mathcal{I}_{\text {albedo. }}$. Under this model, we can compute the appearance loss $\mathcal{L}_{\text {recon }}$ as:

$$
\begin{aligned}
& \mathcal{I}_{\text {recon }}=\mathcal{I}_{\text {albedo }} \odot S\left(\mathcal{N}_{\text {fine }}\right) \\
& \mathcal{L}_{\text {recon }}=\left\|\mathcal{I}_{\text {input }}-\mathcal{I}_{\text {recon }}\right\|_{1}
\end{aligned}
$$




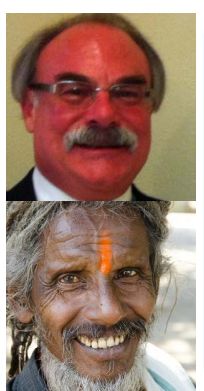

Photograph

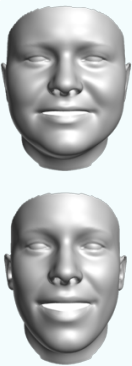

Without Prior

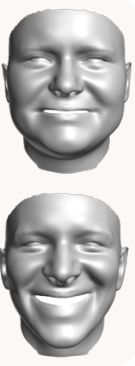

Ours

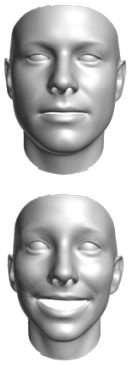

3DDFA

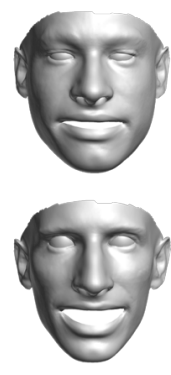

ExpNet

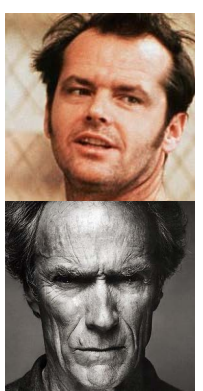

Photograph

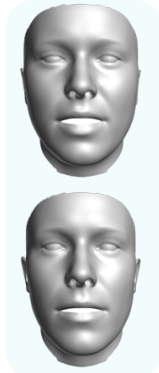

Without Prior

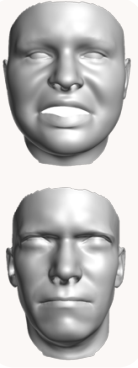

Ours

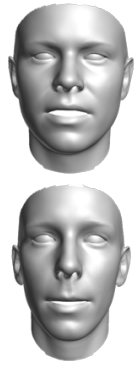

3DDFA

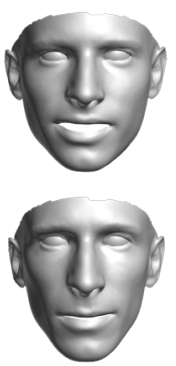

ExpNet

Figure 6. Comparisons of our emotion-driven proxy estimation vs. the state-of-the-art (3DDFA [59] and ExpNet [14])

In order to back propagate $\mathcal{L}_{\text {recon }}$ to update displacement $G(u, v)$, we estimate albedo map $\mathcal{I}_{\text {albedo }}$ and environment lighting $S$ from sparse proxy vertices. Please refer to Section 1 of our supplementary material for detailed algorithm.

For training, we use high resolution facial images from the emotion dataset AffectNet [34], which contains more than $1 \mathrm{M}$ facial images collected from the Internet.

In our experiments, We also use HSV color space instead of RGB to accommodate environment lighting variations and employ a two-step training approach, i.e., only back propagate the loss of PDIM for the first 10 epochs. We find the loss decreases much faster than starting with all losses. Moreover, we train 250 epochs for each facial area. To sum up, our expression estimation and detail synthesis networks borrow the idea of residual learning, breaking down the final target into a few small tasks, which facilitates training and improves performance in our tasks.

\section{Experimental Results}

In order to verify the robustness of our algorithm, we have tested our emotion-driven proxy generation and facial detail synthesis approach on over 20,000 images (see supplementary material for many of these results).

Expression Generation. We downsample all images from AffectNet dataset into $48 \times 48$ (the downsampling is only for proxy generation, not for detail synthesis) and use the Adam optimization framework with a momentum of 0.9 . We train a total of 20 epochs and set learning rate to be 0.001. Our trained Emotion-Net achieves a test accuracy of $52.2 \%$. Recall that facial emotion classification is a challenging task and even human annotators achieve only $60.7 \%$ accuracy. Since our goal focuses on producing more realistic 3D facial models, we find this accuracy is sufficient for producing reasonable expression prior.

Fig. 6 shows some samples of our proxy generation results (without detail synthesis). Compared with the state- of-the-art solutions of 3D expression prediction [59, 14], we find that all methods are able to produce reasonable results in terms of eyes and mouth shape. However, the results from 3DDFA [59] and ExpNet [14] exhibit less similarity with input images on regions such as cheeks, nasolabial folds and under eye bags while ours show significantly better similarity and depict person-specific characteristics. This is because such regions are not covered by facial landmarks. Using landmarks alone falls into the ambiguity mentioned in Section 3.2 and cannot faithfully reconstruct expressions on these regions. Our emotion-based expression predictor exploits global information from images and is able to more accurately capture expressions, especially for jowls and eye bags.

Facial Detail Synthesis. We sample a total of 10K patches for supervised training and $12 \mathrm{~K}$ for unsupervised training. We train 250 epochs in total, and uniformly reduce learning rate from 0.0001 to 0 starting at 100th epoch. Note, we use supervised geometry loss for the first 15 epochs, and then alternate between supervised geometry loss and unsupervised appearance loss for the rest epochs.

Our facial detail synthesis aims to reproduce details from images as realistically as possible. Most existing detail synthesis approaches only rely on illumination and reflectance model [31, 54]. A major drawback of these methods lies in that their synthesized details resemble general object surface without considering skin's spatial correlation, as shown in close-up views in Fig.7(full mesh in supplementary material). Our wrinkles are more similar to real skin surface while the other three approaches are more like cutting with a knife on the surface. We attribute this improvement to combining illumination model with human face statistics from real facial dataset and wrinkle PCA templates.

Our approach also has better performance on handling the surface noise from eyebrows and beards while preserving skin details ( 2 and 6 th row of Fig. 7). 


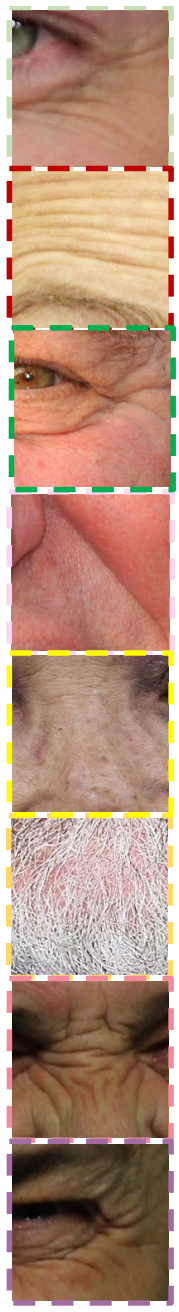

Photograph
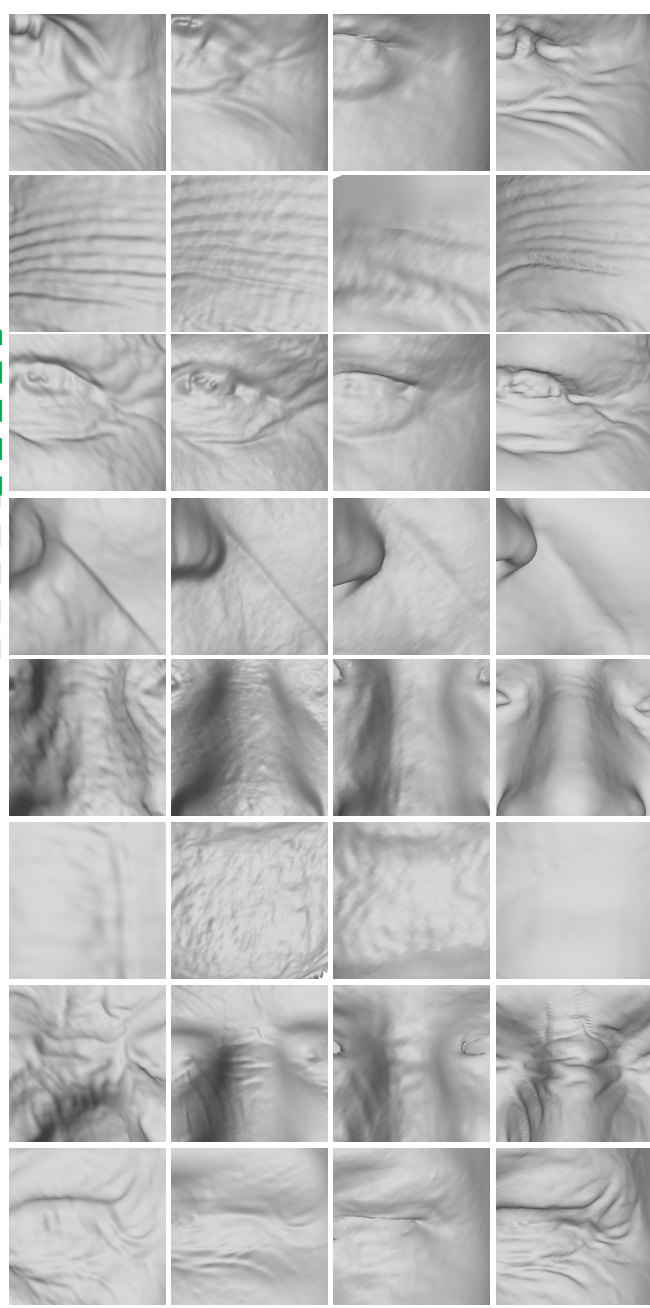

Pix2vertex

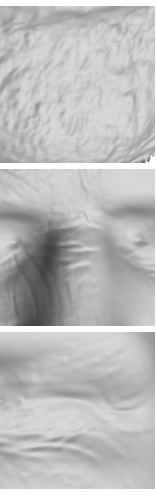

FPD
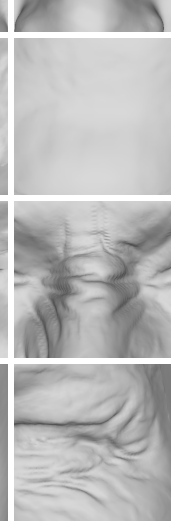

Ours
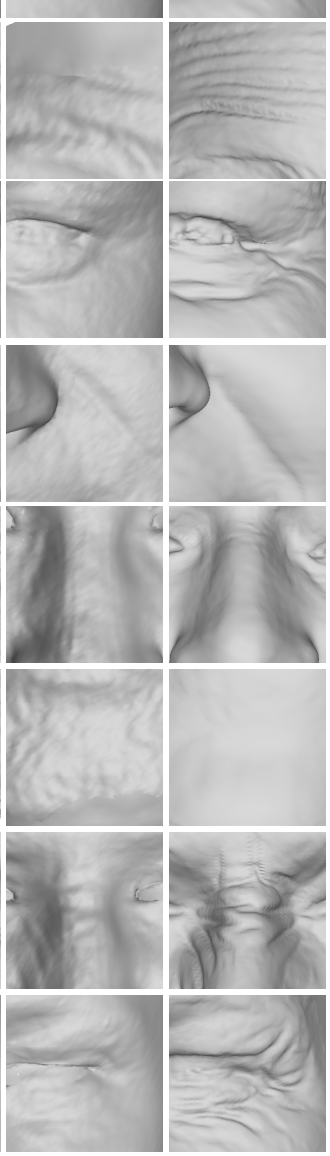

Extreme3D
Figure 7. Close-up views of the synthesized meshes using Pix2vertex [44], FPD [31], Extreme3D [54] and ours.

Quantitative evaluation. We also carry out quantitative comparisons on both proxy mesh and displacement map. Overall, we observe our approach produces much lower errors on strong expressions, especially near the nose and eyebrows where shape deformations are strong. Fig. 8 shows some sample results of proxy + displacement and displacement only errors.

Finally, our output displacement map is easy to integrate with existing rendering pipelines and can produce highfidelity results, as shown in Fig. 1.

\section{Conclusion and Future Work}

We have presented a single-image 3D face synthesis technique that can handle challenging facial expressions while preserving fine geometric structures. Our technique combines cues provided by emotion, expression, appear-

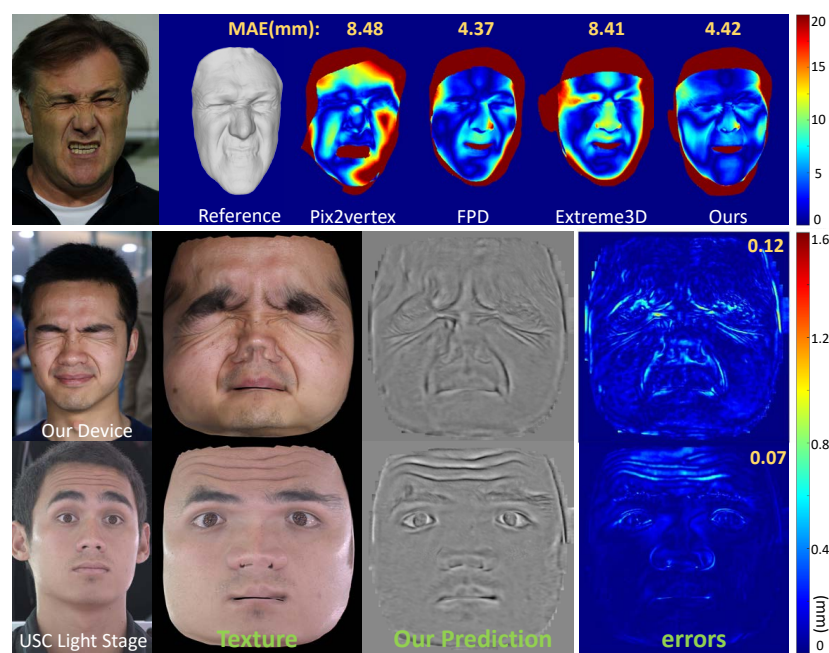

Figure 8. Quantitative comparisons. Top row: the quantitative error maps (proxy + displacement) of Fig. 7 using different methods. Our approach achieves comparable performance on medium to large scale geometry (proxy) but produces much lower error on fine details such as the forehead and nasolabial folds. Two bottom rows: the error map of only the displacement on samples of our capture system and USC LightStage [32].

ance, and lighting for producing high fidelity proxy geometry and fine geometric details. Specifically, we have conducted emotion prediction to obtain an expression-informed proxy and we have demonstrated that our approach can handle a wide range of expressions. For detail synthesis, our Deep Facial Detail Net (DFDN) employs both geometry and appearance loss functions and is trained on real data both captured by our system and from in-the-wild images. Comprehensive experiments have shown that our technique can produce, from a single image, ultra high quality $3 \mathrm{D}$ faces with fine geometric details under various expressions and lighting conditions.

Although our solution is capable of handling a variety of lighting conditions, it has not yet considered the effects caused by occlusions (e.g., hair or glasses), hard shadows that may cause incorrect displacement estimations. For shadows, it may be possible to directly use the proxy to first obtain an ambient occlusion map and then correct the image. Shadow detection itself can be directly integrated into our learning-based framework with new sets of training data. Another limitation of our technique is that it cannot tackle low resolution images: our geometric detail prediction scheme relies heavily on reliable pixel appearance distribution. Two specific types of solutions we plan to investigate are to conduct (learning-based) facial image superresolution that already accounts for lighting and geometric details as our input and to design a new type of proxy face model that includes deformable geometric details. 


\section{References}

[1] Jens Ackermann and Michael Goesele. A survey of photo-

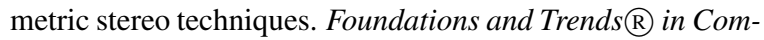
puter Graphics and Vision, 9(3-4):149-254, 2015.

[2] Oleg Alexander, Mike Rogers, William Lambeth, Jen-Yuan Chiang, Wan-Chun Ma, Chuan-Chang Wang, and Paul Debevec. The digital emily project: Achieving a photorealistic digital actor. IEEE Computer Graphics and Applications, 30(4):20-31, 2010.

[3] Daniel G Aliaga and Yi Xu. A self-calibrating method for photogeometric acquisition of 3d objects. IEEE Transactions on Pattern Analysis and Machine Intelligence, 32(4):747754, 2010.

[4] Octavio Arriaga, Matias Valdenegro-Toro, and Paul Plöger. Real-time convolutional neural networks for emotion and gender classification. arXiv preprint arXiv:1710.07557, 2017.

[5] Tadas Baltrušaitis, Marwa Mahmoud, and Peter Robinson. Cross-dataset learning and person-specific normalisation for automatic action unit detection. In 2015 11th IEEE International Conference and Workshops on Automatic Face and Gesture Recognition $(F G)$, volume 6, pages 1-6. IEEE, 2015.

[6] Volker Blanz and Thomas Vetter. A morphable model for the synthesis of $3 \mathrm{~d}$ faces. In Proceedings of the 26th annual conference on Computer graphics and interactive techniques, pages 187-194. ACM Press/Addison-Wesley Publishing Co., 1999.

[7] James Booth, Anastasios Roussos, Allan Ponniah, David Dunaway, and Stefanos Zafeiriou. Large scale 3d morphable models. International Journal of Computer Vision, 126(24):233-254, 2018.

[8] James Booth, Anastasios Roussos, Evangelos Ververas, Epameinondas Antonakos, Stylianos Ploumpis, Yannis Panagakis, and Stefanos Zafeiriou. 3d reconstruction of inthe-wild faces in images and videos. IEEE Transactions on Pattern Analysis and Machine Intelligence, 40(11):26382652, 2018.

[9] Chen Cao, Derek Bradley, Kun Zhou, and Thabo Beeler. Real-time high-fidelity facial performance capture. ACM Transactions on Graphics (ToG), 34(4):46, 2015.

[10] Chen Cao, Qiming Hou, and Kun Zhou. Displaced dynamic expression regression for real-time facial tracking and animation. ACM Transactions on graphics (TOG), 33(4):43, 2014.

[11] Chen Cao, Yanlin Weng, Stephen Lin, and Kun Zhou. 3d shape regression for real-time facial animation. ACM Transactions on Graphics (TOG), 32(4):41, 2013.

[12] Chen Cao, Yanlin Weng, Shun Zhou, Yiying Tong, and Kun Zhou. Facewarehouse: A 3d facial expression database for visual computing. IEEE Transactions on Visualization and Computer Graphics, 20(3):413-425, 2014.

[13] Xuan Cao, Zhang Chen, Anpei Chen, Xin Chen, Shiying Li, and Jingyi Yu. Sparse photometric 3d face reconstruction guided by morphable models. In Proceedings of the IEEE Conference on Computer Vision and Pattern Recognition, pages 4635-4644, 2018.
[14] Feng-Ju Chang, Anh Tuan Tran, Tal Hassner, Iacopo Masi, Ram Nevatia, and Gerard Medioni. Expnet: Landmark-free, deep, 3d facial expressions. In Automatic Face \& Gesture Recognition (FG 2018), 2018 13th IEEE International Conference on, pages 122-129. IEEE, 2018.

[15] Pengfei Dou, Shishir K. Shah, and Ioannis A. Kakadiaris. End-to-end 3d face reconstruction with deep neural networks. 2017 IEEE Conference on Computer Vision and Pattern Recognition (CVPR), pages 1503-1512, 2017.

[16] Carlos Hernandez Esteban, George Vogiatzis, and Roberto Cipolla. Multiview photometric stereo. IEEE Transactions on Pattern Analysis and Machine Intelligence, 30(3):548554, 2008.

[17] Pablo Garrido, Michael Zollhöfer, Dan Casas, Levi Valgaerts, Kiran Varanasi, Patrick Pérez, and Christian Theobalt. Reconstruction of personalized $3 \mathrm{~d}$ face rigs from monocular video. ACM Transactions on Graphics (TOG), 35(3):28, 2016.

[18] Thomas Gerig, Andreas Morel-Forster, Clemens Blumer, Bernhard Egger, Marcel Luthi, Sandro Schönborn, and Thomas Vetter. Morphable face models-an open framework. In Automatic Face \& Gesture Recognition (FG 2018), 2018 13th IEEE International Conference on, pages 75-82. IEEE, 2018.

[19] Abhijeet Ghosh, Graham Fyffe, Borom Tunwattanapong, Jay Busch, Xueming Yu, and Paul Debevec. Multiview face capture using polarized spherical gradient illumination. In ACM Transactions on Graphics (TOG), volume 30, page 129. ACM, 2011.

[20] Paul Graham, Graham Fyffe, Borom Tonwattanapong, Abhijeet Ghosh, and Paul Debevec. Near-instant capture of high-resolution facial geometry and reflectance. In $A C M$ SIGGRAPH 2015 Talks, page 32. ACM, 2015.

[21] Yudong Guo, Juyong Zhang, Jianfei Cai, Boyi Jiang, and Jianmin Zheng. Cnn-based real-time dense face reconstruction with inverse-rendered photo-realistic face images. IEEE Transactions on Pattern Analysis and Machine Intelligence, 2018.

[22] Richard Hartley and Andrew Zisserman. Multiple view geometry in computer vision. Cambridge university press, 2003.

[23] Guosheng Hu, Fei Yan, Josef Kittler, William Christmas, Chi Ho Chan, Zhenhua Feng, and Patrik Huber. Efficient 3d morphable face model fitting. Pattern Recognition, 67:366379, 2017.

[24] Patrik Huber, Guosheng Hu, Rafael Tena, Pouria Mortazavian, P Koppen, William J Christmas, Matthias Ratsch, and Josef Kittler. A multiresolution 3d morphable face model and fitting framework. In Proceedings of the 11th International Joint Conference on Computer Vision, Imaging and Computer Graphics Theory and Applications, 2016.

[25] Loc Huynh, Weikai Chen, Shunsuke Saito, Jun Xing, Koki Nagano, Andrew Jones, Paul Debevec, and Hao Li. Mesoscopic facial geometry inference using deep neural networks. In Proceedings of the IEEE Conference on Computer Vision and Pattern Recognition, pages 8407-8416, 2018.

[26] Aaron S. Jackson, Adrian Bulat, Vasileios Argyriou, and Georgios Tzimiropoulos. Large pose $3 \mathrm{~d}$ face reconstruc- 
tion from a single image via direct volumetric cnn regression. 2017 IEEE International Conference on Computer Vision (ICCV), pages 1031-1039, 2017.

[27] Luo Jiang, Juyong Zhang, Bailin Deng, Hao Li, and Ligang Liu. 3d face reconstruction with geometry details from a single image. IEEE Transactions on Image Processing, 27(10):4756-4770, 2018.

[28] Ira Kemelmacher-Shlizerman and Ronen Basri. 3d face reconstruction from a single image using a single reference face shape. IEEE transactions on pattern analysis and machine intelligence, 33(2):394-405, 2011.

[29] Hyeongwoo Kim, Michael Zollhöfer, Ayush Tewari, Justus Thies, Christian Richardt, and Christian Theobalt. Inversefacenet: Deep monocular inverse face rendering. In Proceedings of the IEEE Conference on Computer Vision and Pattern Recognition, pages 4625-4634, 2018.

[30] Tianye Li, Timo Bolkart, Michael J Black, Hao Li, and Javier Romero. Learning a model of facial shape and expression from 4d scans. ACM Transactions on Graphics (TOG), 36(6):194, 2017.

[31] Yue Li, Liqian Ma, Haoqiang Fan, and Kenny Mitchell. Feature-preserving detailed $3 \mathrm{~d}$ face reconstruction from a single image. In Proc. of the 15th ACM SIGGRAPH European Conference on Visual Media Production. ACM, 2018.

[32] Wan-Chun Ma, Tim Hawkins, Pieter Peers, Charles-Felix Chabert, Malte Weiss, and Paul Debevec. Rapid acquisition of specular and diffuse normal maps from polarized spherical gradient illumination. In Proceedings of the 18th Eurographics conference on Rendering Techniques, pages 183194. Eurographics Association, 2007.

[33] Jiri Matas, Ondrej Chum, Martin Urban, and Tomás Pajdla. Robust wide-baseline stereo from maximally stable extremal regions. Image and vision computing, 22(10):761767, 2004.

[34] Ali Mollahosseini, Behzad Hasani, and Mohammad H Mahoor. Affectnet: A database for facial expression, valence, and arousal computing in the wild. arXiv preprint arXiv:1708.03985, 2017.

[35] Thoma Papadhimitri and Paolo Favaro. A new perspective on uncalibrated photometric stereo. In Proceedings of the IEEE Conference on Computer Vision and Pattern Recognition, pages 1474-1481, 2013.

[36] Jaesik Park, Sudipta N. Sinha, Yasuyuki Matsushita, YuWing Tai, and In-So Kweon. Robust multiview photometric stereo using planar mesh parameterization. IEEE Transactions on Pattern Analysis and Machine Intelligence, 39:1591-1604, 2017.

[37] Pascal Paysan, Reinhard Knothe, Brian Amberg, Sami Romdhani, and Thomas Vetter. A 3d face model for pose and illumination invariant face recognition. In Advanced video and signal based surveillance, 2009. AVSS'09. Sixth IEEE International Conference on, pages 296-301. Ieee, 2009.

[38] Yvain Quéau. Reconstruction tridimensionnelle par stéréophotométrie. $\mathrm{PhD}$ thesis, 2015.

[39] Elad Richardson, Matan Sela, and Ron Kimmel. 3d face reconstruction by learning from synthetic data. In $3 D$ Vision $(3 D V), 2016$ Fourth International Conference on, pages 460469. IEEE, 2016.
[40] Elad Richardson, Matan Sela, Roy Or-El, and Ron Kimmel. Learning detailed face reconstruction from a single image. In Computer Vision and Pattern Recognition (CVPR), 2017 IEEE Conference on, pages 5553-5562. IEEE, 2017.

[41] Sami Romdhani and Thomas Vetter. Estimating 3d shape and texture using pixel intensity, edges, specular highlights, texture constraints and a prior. In Computer Vision and Pattern Recognition, 2005. CVPR 2005. IEEE Computer Society Conference on, volume 2, pages 986-993. IEEE, 2005.

[42] Shunsuke Saito, Tianye Li, and Hao Li. Real-time facial segmentation and performance capture from rgb input. In European Conference on Computer Vision, pages 244-261. Springer, 2016.

[43] Sandro Schönborn, Bernhard Egger, Andreas Morel-Forster, and Thomas Vetter. Markov chain monte carlo for automated face image analysis. International Journal of Computer Vision, 123(2):160-183, 2017.

[44] Matan Sela, Elad Richardson, and Ron Kimmel. Unrestricted facial geometry reconstruction using image-to-image translation. In 2017 IEEE International Conference on Computer Vision (ICCV), pages 1585-1594. IEEE, 2017.

[45] Soumyadip Sengupta, Angjoo Kanazawa, Carlos D. Castillo, and David W. Jacobs. Sfsnet: Learning shape, reflectance and illuminance of faces 'in the wild'. 2018 IEEE/CVF Conference on Computer Vision and Pattern Recognition, pages 6296-6305, 2017.

[46] Fuhao Shi, Hsiang-Tao Wu, Xin Tong, and Jinxiang Chai. Automatic acquisition of high-fidelity facial performances using monocular videos. ACM Transactions on Graphics (TOG), 33(6):222, 2014.

[47] Giota Stratou, Abhijeet Ghosh, Paul Debevec, and LouisPhilippe Morency. Effect of illumination on automatic expression recognition: a novel $3 \mathrm{~d}$ relightable facial database. In Automatic Face \& Gesture Recognition and Workshops (FG 2011), 2011 IEEE International Conference on, pages 611-618. IEEE, 2011.

[48] Ariel Tankus and Nahum Kiryati. Photometric stereo under perspective projection. In Computer Vision, 2005. ICCV 2005. Tenth IEEE International Conference on, volume 1, pages 611-616. IEEE, 2005.

[49] Ayush Tewari, Michael Zollhöfer, Pablo Garrido, Florian Bernard, Hyeongwoo Kim, Patrick Pérez, and Christian Theobalt. Self-supervised multi-level face model learning for monocular reconstruction at over $250 \mathrm{hz}$. In Proceedings of the IEEE Conference on Computer Vision and Pattern Recognition, pages 2549-2559, 2018.

[50] Ayush Tewari, Michael Zollhöfer, Hyeongwoo Kim, Pablo Garrido, Florian Bernard, Patrick Pérez, and Christian Theobalt. Mofa: Model-based deep convolutional face autoencoder for unsupervised monocular reconstruction. In The IEEE International Conference on Computer Vision (ICCV), volume 2, page 5, 2017.

[51] Justus Thies, Michael Zollhofer, Marc Stamminger, Christian Theobalt, and Matthias Nießner. Face2face: Real-time face capture and reenactment of rgb videos. In Proceedings of the IEEE Conference on Computer Vision and Pattern Recognition, pages 2387-2395, 2016. 
[52] Anh Tuan Tran, Tal Hassner, Iacopo Masi, and Gérard Medioni. Regressing robust and discriminative 3d morphable models with a very deep neural network. In Computer Vision and Pattern Recognition (CVPR), 2017 IEEE Conference on, pages 1493-1502. IEEE, 2017.

[53] Anh Tuan Tran, Tal Hassner, Iacopo Masi, Eran Paz, Yuval Nirkin, and Gérard Medioni. Extreme 3D face reconstruction: Seeing through occlusions. In IEEE Conf. on Computer Vision and Pattern Recognition (CVPR), 2018.

[54] Anh Tuân Tran, Tal Hassner, Iacopo Masi, Eran Paz, Yuval Nirkin, and Gérard Medioni. Extreme 3d face reconstruction: Seeing through occlusions. In Proc. CVPR, 2018.

[55] Daniel Vlasic, Matthew Brand, Hanspeter Pfister, and Jovan Popović. Face transfer with multilinear models. ACM transactions on graphics (TOG), 24(3):426-433, 2005.

[56] Matthew J. Westoby, James Brasington, Neil F. Glasser, Michael John Hambrey, and Jennifer M. Reynolds. 'structure-from-motion' photogrammetry: A low-cost, effective tool for geoscience applications. 2012.

[57] Chenglei Wu, Yebin Liu, Qionghai Dai, and Bennett Wilburn. Fusing multiview and photometric stereo for $3 \mathrm{~d}$ reconstruction under uncalibrated illumination. IEEE transactions on visualization and computer graphics, 17(8):10821095, 2011.

[58] Lijun Yin, Xiaozhou Wei, Yi Sun, Jun Wang, and Matthew J. Rosato. A 3d facial expression database for facial behavior research. 7th International Conference on Automatic Face and Gesture Recognition (FGR06), pages 211-216, 2006.

[59] Xiangyu Zhu, Zhen Lei, Xiaoming Liu, Hailin Shi, and Stan Z. Li. Face alignment across large poses: A 3d solution. 2016 IEEE Conference on Computer Vision and Pattern Recognition (CVPR), pages 146-155, 2016.

[60] Xiangyu Zhu, Junjie Yan, Dong Yi, Zhen Lei, and Stan Z. Li. Discriminative 3d morphable model fitting. 2015 11th IEEE International Conference and Workshops on Automatic Face and Gesture Recognition (FG), 1:1-8, 2015. 